\title{
Data Materiality
}

\author{
David Bouchard \\ Ryerson University \\ Toronto, Canada \\ david.bouchard@ryerson.ca
}

\section{ABSTRACT}

This demonstration develops a series of illustrated case studies based on data-driven artworks developed by the author in the last five years. The artworks all examine the notion of using data as a raw material that can be filtered, manipulated or moulded into representations which suggest an experience of the data, rather than an understanding of it. The preoccupation of these works isn't finding meaning or answers in the data, but rather to evoke impressions in the viewer, to provide alternative perspectives, or perhaps pose new questions. Aesthetic considerations are also derived from the unique nature of each individual dataset, in the same way one would engage with any other type of raw material. The interactive presentation will reflect on and situate these works according to theories, ideas and paradoxes within the current discourse on data art, for instance data as anti-information, anti-sublime, as an immaterial material and or as a found object.

\section{SUMMARY OF THE PROJECTS}

\subsection{Tomographic perspectives}

This project was a collaboration with $\mathrm{Dr}$ Beau Standish and Dr Adrian Mariampillai, researchers at Ryerson University in the Department of Electrical and Computer Engineering. We used 3dimensional volumetric images depicting blood vessels in cancerous tissues as the basis for the generation of abstract visuals and video loops. Using image processing techniques, we extracted structural information from the vessels. These structures then became starting points for the creation of images inspired by the organic qualities of the blood vessels themselves.

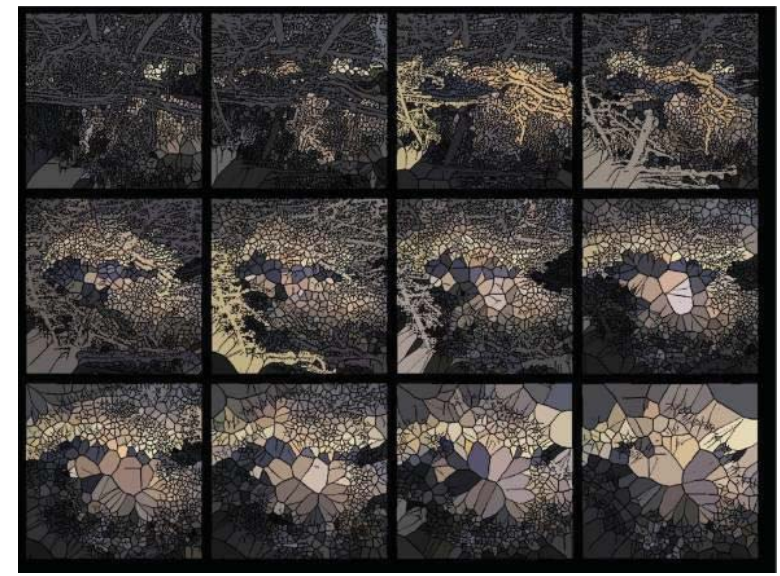

Figure 1: Tomographic Perspectives (2010)

\subsection{Observation instruments}

Observation Instruments introduces two interfaces, which enable the viewer to interact with time-lapsed projections of a webcam image from Kimmirut, Nunavut. The images are pulled from a database, collected by the artist over a period of two years and consisting of approximately 65,000 images of May 2012. The database also includes weather statistics at the time the image was recorded. The instruments provide the viewer with an opportunity to re-imagine the remote site and shape their interpretations according to time and weather parameters.

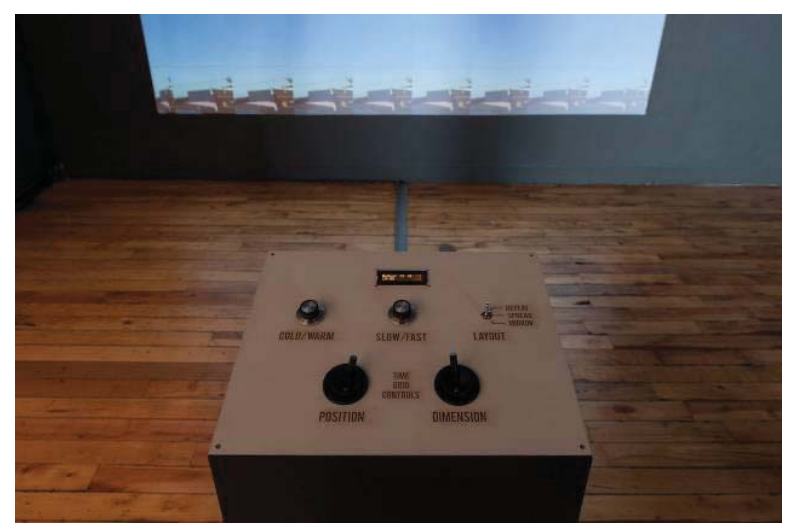

Figure 2: Observation Instruments (2011) 


\subsection{One year in kimmirut}

One Year in Kimmirut is a piece derived from the same database of Kimmirut webcam images. The series of prints visualize one year of time-lapsed photographs archived at 15-minute intervals. Each panel represents one month of the year, from January to December. Each column of photographs represents a single day.

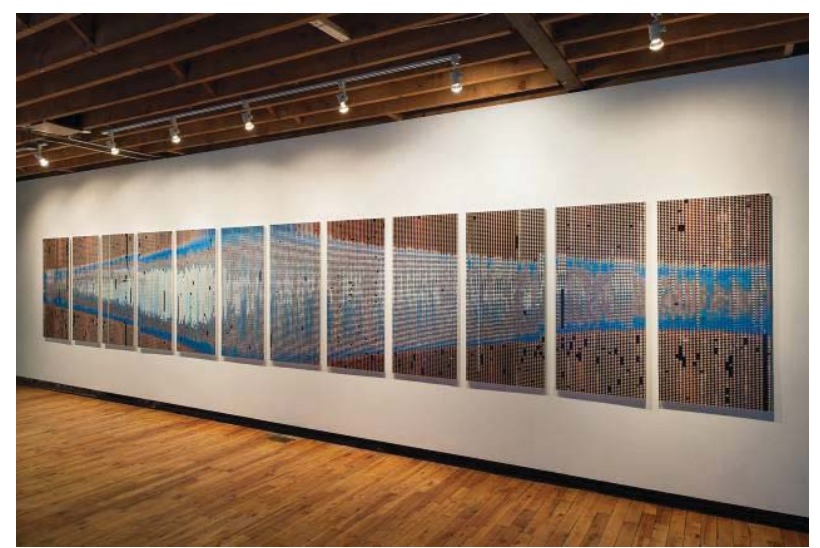

Figure 3: One Year in Kimmirut (2011)

\subsection{Transients}

Transients is a series of generative animations inspired by the notions of flow, ephemerality and transitory states. The underlying structure of these animations is a database created using GPS data from the Toronto public transit system. The data, available on the web through the City of Toronto Open Data portal, includes the location, routes and stops of every bus and streetcar in the system, as well as the arrival times of trains within underground subway stations. The software establishes an aesthetic framework for the data to unfold within, balancing artistic and algorithmic decisions alongside existing patterns within the data. The project was presented to commuters on screens within the transit system.

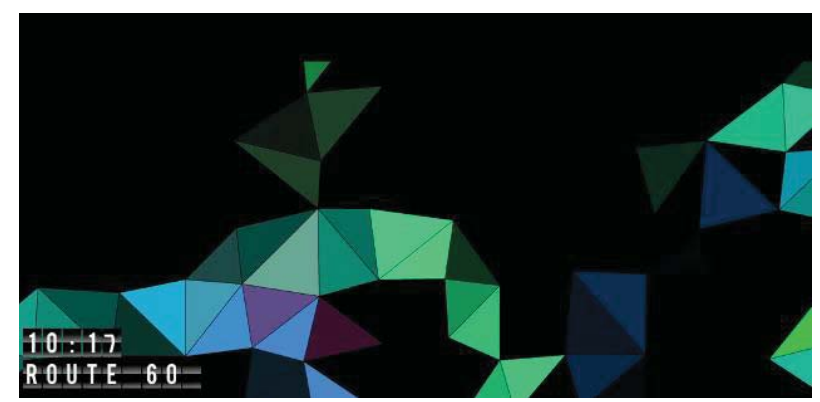

Figure 4: Transients (2012)

\subsection{Urban infosonics}

Urban Infosonics is a work currently in development, which seeks out the aestheticization and creative transformation of public data. A database built from the City Council minutes and councillor voting records is used as the raw material for a multichannel, interactive sound installation. This work explores the relationship between terms commonly used in sound art, such as resonance and reverberation, consonance and dissonance, and which also find application in more social discourse and political commentary. Can the similarities between the definitions of these concepts be used as template for mapping specific pieces of information or metadata into specific sonic parameters?

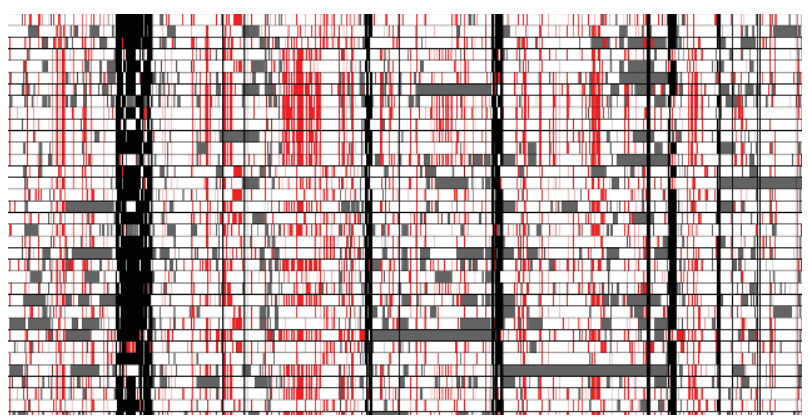

Figure 5: Urban Infosonics (2014, ongoing)

\section{BIBIOGRAPHY}

Wanner, A. and Beer, R. (2013) Found data: generating natural looking shapes by appropriating scientific data, Proceedings of the $1^{\text {st }}$ Conference on Computation, Communication, Aesthetics and the $X$, Bergamo, Italy.

Diamond, S. (2011) Data visualization: materiality and mediation, International Symposium on Electronic Arts, Istanbul, Turkey.

Whitelaw, M. (2008) Art against information: case studies in data practice. Fibre Culture Journal, issue 11.

Viegas, F and Wattenberg, M. (2007) Artistic data visualization: beyond visual analytics, Proceedings of the $2^{\text {nd }}$ International Conference on Online Communities and Social Computing, pp. 182-191, Bejing, China.

Manovich, L. (2002) The Anti-Sublime Ideal in Data Art, http://www.manovich.net/DOCS/data art.doc (accessed 2014). 